Nota de investigación

\title{
Sistema computacional bajo la metodología PADPEEM para estimar carbono aéreo en café
}

\author{
Saira Edith Márquez de la Cruz ${ }^{1}$ \\ José Luis García Cué ${ }^{1 \S}$ \\ Yolanda M. Fernández Ordóñez ${ }^{1}$ \\ Juan Ángel Tinoco Rueda ${ }^{2}$ \\ David H. Del Valle Paniagua ${ }^{1}$ \\ Dora Ma. Sangermán-Jarquín ${ }^{3}$ \\ ${ }^{1}$ Colegio de Postgraduados en Ciencias Agrícolas. Carretera México-Texcoco km 36.5, Montecillo, \\ Texcoco, Estado de México. CP. 56230. Tel. 55 58045900. (marquez.saira@colpos.mx; \\ yfernand@colpos.mx; dhvallep@colpos.mx). ${ }^{2}$ Universidad Autónoma Chapingo-Centro Regional \\ Universitario Oriente. Carretera Federal Huatusco-Xalapa km 6.5, Huatusco, Veracruz. CP. 94100. \\ (tinoco@correo.chapingo.mx). ${ }^{3}$ Campo Experimental Valle de México-INIFAP. Carretera los Reyes- \\ Texcoco km 13.5. Coatlinchán, Texcoco, Estado de México, México. CP. 56250. Tel. 595921268. \\ (sangerman.dora@inifap.gob.mx). \\ ${ }^{\S}$ Autor para correspondencia: jlgcue@ colpos.mx.
}

\section{Resumen}

La investigación tuvo por objetivo diseñar un sistema computacional Web-App para el registro de datos in situ y para la estimación del almacenamiento de carbono aéreo en parcelas de café en Huatusco, Veracruz, México. Lo anterior se planteó por las necesidades de los productores para automatizar los cálculos de captura de $\mathrm{CO}_{2}$ que sirven en la contribución de la mitigación del cambio climático. El software se elaboró en el Campus Montecillo del Colegio de Postgraduados de febrero a octubre de 2020 a través de la propuesta de la metodología PADPEEM y probado con los datos obtenidos en algunos cultivos de café en Huatusco en enero de 2020. En los resultados se mostró el sistema CarbonoCafé, su arquitectura, mapa de navegación e interfaces. El sistema CarbonoCafé será de impacto en la reducción de recursos humanos, económicos y de tiempo, para el cálculo de carbono aéreo in situ. Además, contiene bases de datos que se pueden consultar desde cualquier computadora o teléfono móvil.

Palabras clave: App, mitigación del cambio climático, sistema web.

Recibido: agosto de 2021

Aceptado: septiembre de 2021 
Bazán et al. (2017) explicaron que en los últimos años el uso de las tecnologías de la información y la comunicación (TIC) han pasado a formar parte de la vida cotidiana de un gran número de personas, lo que ha provocado en los usuarios la necesidad de contar con software y aplicaciones (App) que les permitan interactuar para el intercambio de contenidos, educación, investigación, uso de servicios, tecnificación agrícola y la colaboración en diversas tareas conjuntas desde distintos sitios.

Bakht (2018) expresó que lo anterior no se hubiera logrado sin el desarrollo de la computación distribuida a través de las redes de computadoras, páginas web, sistemas multiplataforma y aplicaciones (App) que funcionan con diferentes sistemas operativos y que pueden interconectarse entre si. En la actualidad, hay diverso software que permite el levantamiento de información en campo a través de dispositivos móviles facilitando el trabajo a investigadores y otro tipo de profesionistas que requieren de estas labores.

Dichos sistemas coadyuvan en diversas áreas del conocimiento, en especial en aquellas de apoyo de servicios agroforestales y ambientales enfocadas a la mitigación de los efectos del cambio climático provocados principalmente por la acumulación de moléculas de dióxido de carbono $\left(\mathrm{CO}_{2}\right)$. En la búsqueda de esos sistemas, se buscó software y App que trabajan con información agroforestal, captura de carbono aéreo y biomasa in situ, pero se detectó escasa información. Más adelante, se realizó una revisión de cultivos que ayuden a mitigar el efecto invernadero y que puedan captar el $\mathrm{CO}_{2}$.

De acuerdo con lo sugerido por Isaza y Cornejo (2015) se eligió el café porque capta una gran cantidad de la molécula, además, por su interés comercial, social y cultural. Después, se buscó una zona cafetalera en México y se destacó a la región de Huatusco en el estado de Veracruz como una de las zonas de mayor volumen agroforestal con producción bajo sombra. Otro aspecto que fue considerado para la elección de la región es porque se puede tener acceso a las parcelas de los productores que apoyan a investigadores de instituciones educativas de educación superior y que están participando para la mejora en sus cosechas.

Para la estimación de carbono almacenado en cafetales hay diversas metodologías, pero se resaltan las de Tinoco y Escamilla (2017) y Rojas-García et al. (2015) que lo hacen considerando cinco reservorios: perennes de sombra, cafetos, hojarasca, herbáceas y suelo. La forma de evaluar las perennes de sombra y cafetos es mediante la medición del diámetro normal y la altura total de los individuos, y posteriormente se aplican ecuaciones alométricas para relacionar esas variables con el peso seco de la biomasa vegetal. Con respecto a los reservorios de hojarasca, herbáceas y suelo se recolectan muestras para analizarlas en laboratorio.

Todo este proceso lo realizan investigadores, profesores, estudiantes y productores recolectando datos in situ lo que provoca una gran inversión de tiempo para hacerlo porque se hace de manera manual llenando diferentes formatos. Después, la información se captura en hojas de cálculo y se aplican las diferentes fórmulas alométricas. El proceso anterior puede provocar errores desde el registro de información, la captura de datos, hasta los resultados por mal aplicación de fórmulas alométricas. Por tal motivo, se pensó en un software que coadyuve a la realización de todas estas actividades y que además tenga la capacidad de tomar datos en campo a través de dispositivos móviles y que queden registrados en bases de datos. 
Para la construcción del software, se investigó sobre aplicaciones distribuidas a través de redes de computadoras. Bazán et al. (2017) explicaron que estas redes, trabajan bajo el modelo clienteservidor apoyados del protocolo de control de transmisión/protocolo de internet (TCP/IP), que permite la comunicación de equipos de escritorio y móviles, con diferentes sistemas operativos, conectados a la red. Las aplicaciones se diseñan bajo una arquitectura Web-App siguiendo diferentes estructuras como la $\mathrm{LAMJ}+\mathrm{G}$ por sus siglas de un servidor web utilizando el Sistema Operativo Linux, Servidor Apache, gestor de bases de datos MySQL, JavaScript y tecnologías Geomáticas bajo QGis (GNU-General Public License) y mapas dinámicos.

Por todo lo anterior, se llegó a la siguiente pregunta ¿qué metodología híbrida puede usarse para el diseño de un sistema informático que registre datos in situ de vegetación, suelo y de indicadores para el diagnóstico de la estructura productiva de parcelas de café y que además estime la captura de carbono aéreo en agroecosistemas de café en Huatusco, Veracruz, México? Para contestar la pregunta se propuso una investigación para diseñar un sistema computacional Web-App para el registro de datos in situ y la estimación del almacenamiento de carbono aéreo en parcelas de café en Huatusco, Veracruz, México.

La hipótesis propuesta fue que el desarrollo de un sistema informático apoyado de una metodología híbrida permite registrar datos in situ de plantas, árboles y cafetos, así como la estimación del almacenamiento de carbono orgánico aéreo en agroecosistemas en la zona. Para el desarrollo del sistema se propuso utilizar herramientas de software libre e Internet que permitan registrar datos en campo y estimar en tiempo real la captura de carbono aéreo en sistemas agroforestales de café de la región de Huatusco, Veracruz, México y que además pueda ser utilizado por académicos, alumnos, especialistas forestales, productores, entre otros. Asimismo, que maneje bases de datos y que genere reportes a través de documentos, información en pantalla o en mapas dinámicos.

El sistema se prevé que tenga un alcance para la región cafetalera de Huatusco. Una limitación es que el software solo funciona para la región seleccionada, la razón es que para otra zona geográfica distinta se tienen que hacer adecuaciones en la programación de los algoritmos de fórmulas alométricas de acuerdo con las características de cada región y de los agroecosistemas para el cálculo de captura de carbono aéreo.

Para contestar la pregunta de investigación y cumplir con el objetivo se planteó como parte de los materiales y métodos la construcción del sistema computacional apoyándose de buenas prácticas de Ingeniería de Software, la metodología PADDIEM (Meráz et al., 2019) y la metodología Mobile-D (Amaya, 2013).

PADDIEM se utilizó para diseño de software educativo y constó de siete diferentes fases: planeación, análisis, diseño, desarrollo, implementación, evaluación y mantenimiento. Mobile-D se basó en diferentes modelos ágiles como el de programación extrema (XP) y su finalidad principal fue obtener ciclos de desarrollos muy rápidos para que el paquete informático esté terminado en poco tiempo, constó de cinco etapas: exploración, inicialización, producción, estabilización y pruebas del sistema.

Más adelante, se vio la necesidad de proponer una metodología híbrida que integrara tanto a PADDIEM como a Mobile-D en el desarrollo del sistema. La razón es que las características de cada una, por separado, no se ajustaban a lo que se pretende en este proyecto. Del PADDIEM se 
consideraron las fases de planeación, análisis, evaluación y mantenimiento. De Mobile-D se consideraron las de producción y estabilización que permiten el desarrollo de pequeños productos de manera rápida y la liberación de pequeños segmentos del sistema evaluando su funcionamiento. La metodología resultante tomó el nombre de PADPEEM. El diagrama de flujo de la Figura 1 muestra la metodología propuesta donde se destaca cada etapa por rectángulos y las diferentes evaluaciones por rombos.

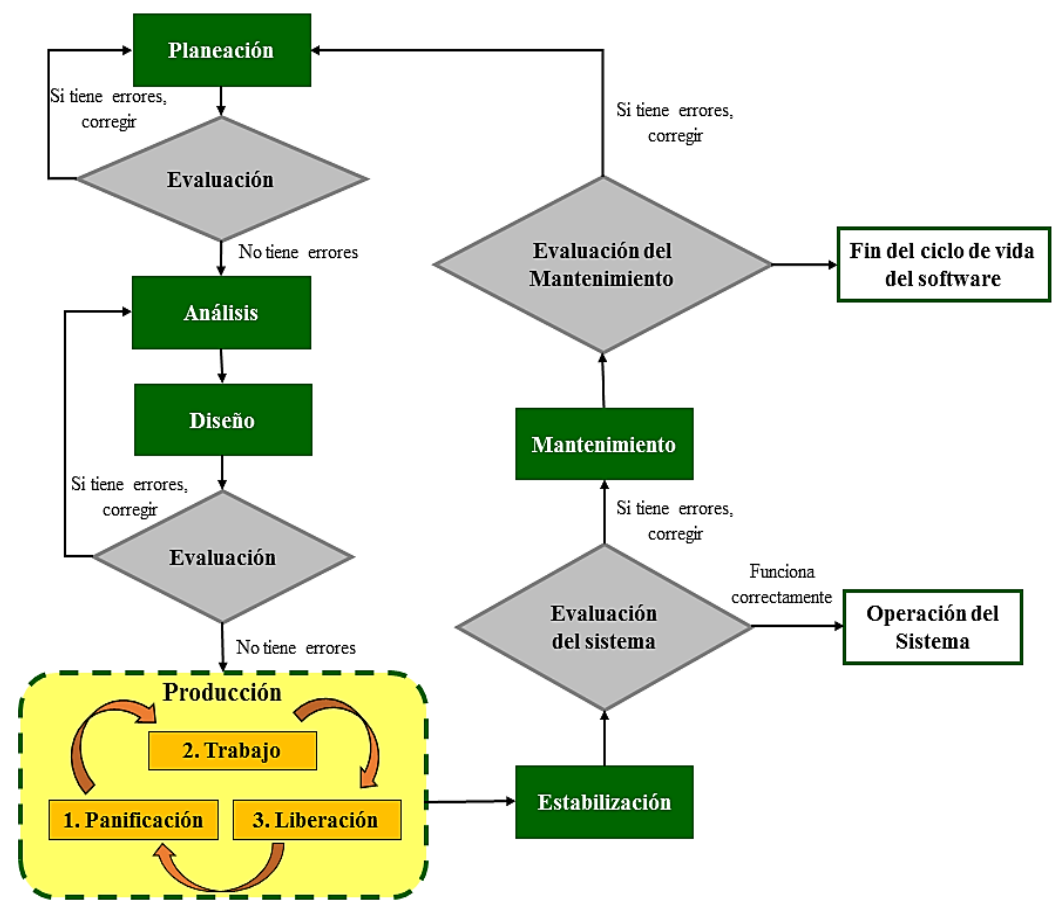

\section{Figura 1. Propuesta de metodología híbrida PADPEEM.}

Después de elegir la metodología PADPEEM se puso en marcha el desarrollo del software que se programó en el Campus Montecillo del Colegio de Postgraduados de febrero a octubre de 2020. Se siguió cada etapa del PADPEEM como se explica a continuación:

\section{Planeación}

Se identificó la necesidad del proyecto que fue el diseño de un sistema computacional para el registro de datos in situ y la estimación del almacenamiento de carbono aéreo a través de las metodologías de Tinoco y Escamilla (2017) así como la de Rojas-García et al. (2015) en agroecosistemas de café en Huatusco, Veracruz. Más adelante, se realizó un estudio de viabilidad del proyecto mediante el análisis de las fortalezas, oportunidades, debilidades y amenazas (FODA).

Posterior a esto, se identificaron los recursos económicos que se tenían disponibles tanto para el hardware como para el software. Además, se tomó en cuenta el tiempo para la realización del sistema. También, se propusieron los requerimientos tecnológicos y los humanos estableciendo equipos multidisciplinarios y las actividades a realizar en cada etapa de PADPEEM (Figura 2). Asimismo, se hizo un cronograma de actividades. 


\begin{tabular}{|c|c|c|}
\hline $\begin{array}{c}\text { Equipos multidisciplinarios } \\
\text { de expertos }\end{array}$ & Etapas & Actividades a realizar \\
\hline $\begin{array}{l}\text { Equipo de cómputo } \\
\text { Equipo de sustentabilidad } \\
\text { en café } \\
\text { Equipo de diseño gráfico }\end{array}$ & $\mathbf{P}$ & $\begin{array}{l}\text { 1. Problemática y Necesidades } \\
\text { 2. Propósito } \\
\text { 3. Estudio de viabilidad } \\
\text { 4. Requerimientos tecnológicos y humanos } \\
\text { 5. Cronograma }\end{array}$ \\
\hline $\begin{array}{l}\text { *quipo de cómputo } \\
\text { Equipo de sustentabilidad } \\
\text { en café } \\
\text { Equipo de diseño gráfico }\end{array}$ & $\mathbf{A}$ & $\begin{array}{l}\text { 1. Propuesta del sistema } \\
\text { 2. Ámbito del sistema } \\
\text { 3. Especificación general tanto de usuarios como de los } \\
\text { requisitos del software } \\
\text { 4. Especificación detallada de los requerimientos del } \\
\text { sistema. } \\
\text { 5. Elección de la arquitectura, tipo de aplicación, lenguajes } \\
\text { de programación y software a utilizar }\end{array}$ \\
\hline $\begin{array}{l}\text { * Equipo de cómputo } \\
\text { Equipo de diseño gráfico }\end{array}$ & D & $\begin{array}{l}\text { 1. Arquitectura general del sistema. } \\
\text { 2. Mapa de navegación y permisos de usuarios } \\
\text { 3. Propuesta de base de datos } \\
\text { 4. Propuesta de diseño de interfaces. }\end{array}$ \\
\hline * Equipo de cómputo & & $\begin{array}{l}\text { 1. Creación del servidor web local } \\
\text { 2. Elaboración de base de datos } \\
\text { 3. Elaboración de interfaces } \\
\text { 4. Programación de algoritmos para la estimación de } \\
\text { biomasa y carbono } \\
\text { 5. Generación de reportes }\end{array}$ \\
\hline * Equipo de cómputo & E & $\begin{array}{l}\text { 1. Alojamiento de la base de datos en un servidor web } \\
\text { remoto } \\
\text { 2. Construcción de metadatos } \\
\text { 3. Primera versión del sistema } \\
\text { 4. Instalación } \\
\text { 5. Pruebas piloto } \\
\text { 6. Capacitación para el uso del sistema } \\
\end{array}$ \\
\hline $\begin{array}{l}\text { Equipo de cómputo } \\
\text { Equipo de sustentabilidad } \\
\text { en café } \\
\text { Equipo de diseño gráfico }\end{array}$ & 12 & $\begin{array}{l}\text { 1. Evahuación de la etapa de Planeación } \\
\text { 2. Evaluación de la etapa de Análisis y Diseño } \\
\text { 3. Evahuación de la etapa de Producción y Estabilización } \\
\text { 4. Evahuación de la opinión de los usuarios } \\
\text { 5. Evaluación de la etapa de Mantenimiento }\end{array}$ \\
\hline $\begin{array}{l}\text { Equipo de cómputo } \\
\text { Equipo de diseño gráfico }\end{array}$ & & $\begin{array}{l}\text { 1. Corrección de errores detectados en la evaluación de cada } \\
\text { etapa } \\
\text { 2. Mejorar la implementación de las unidades del sistema } \\
\text { 3. Incrementar los servicios del sistema conforme se } \\
\text { descubren nuevos requerimientos. } \\
\text { 4. Eliminar el sistema cuando finalice su ciclo de vida }\end{array}$ \\
\hline
\end{tabular}

Figura 2. Equipos multidisciplinarios y su participación en cada etapa de PADPEEM.

\section{Análisis}

En esta etapa se hizo la propuesta del sistema, el ámbito, especificaciones generales, requerimientos, selección de arquitectura, tipo de aplicación y software a utilizar. Para cumplir con el propósito del software se propusieron dos tipos de aplicaciones, una web y otra móvil híbrida (Web-App). También, se establecieron seis tipos de usuarios que pueden acceder al sistema: administrador, investigador, técnico especializado, estudiante, apoyo de técnico especializado y productor. Cada uno de ellos con un permiso o rol específico para ingresar al sistema y acceder a ciertas vistas.

Además, el sistema se proyectó con seis módulos principales ubicados en el menú de la pantalla principal: guía del usuario, gestión de usuario, gestión de catálogos, planeación de muestreo, muestreo en campo y reportes. Asimismo, se detectó la necesidad de buscar un servicio de 
hospedaje web remoto que se ajustara a la estructura LAMP+G para alojar las bases de datos y software a utilizar, debido a que la laptop con la que se contaba no cumplía con los requerimientos tecnológicos necesarios y no contaba con una dirección de internet fija (dominio). Por tal razón, se contrató el servicio de hospedaje 'hospedando.mx' que cumplía con los requisitos para el proyecto.

\section{Diseño}

Se planteó la estructura de las bases de datos: una relacional y otras llamadas NoSQL. También, se proyectaron los diferentes interfaces y sus diferentes componentes para la App a través de Visual Studio Code (Massachusetts Institute of Technology, License) así como los lenguajes de programación JavaScript, CSS, HTML y React Native. Más adelante, se diseñaron los algoritmos que proporcionaron los cálculos para la estimación de biomasa y captura de carbono aéreo por medio de las ecuaciones alométricas de Rojas-García et al. (2015), en especial las especies más representativas de las parcelas de café de esa zona.

Además, se consideró que la App pueda almacenar información en archivos JSON dentro del teléfono móvil y puede crear reportes en archivos PDF (Acrobat license) o en un mapa dinámico. Asimismo, se tomó en cuenta que en el sitio web se puede obtener información a través de un sistema gestor de bases de datos o en un sistema de información geográfico como QGis.

\section{Producción}

De manera remota, dentro del servidor web contratado, se desarrolló la base de datos relacional, para la cual se utilizó el sistema gestor de base de datos MySQL y el lenguaje SQL que incluye datos generales del predio, muestreo y su georreferenciación. Además, en el equipo de cómputo con el que se contaba (laptop con Intel Core i5, 8 RAM, 1 TB de disco duro), se desarrolló una base de datos NoSQL utilizando el lenguaje de programación JavaScript y el almacenamiento AsyncStorage del marco de trabajo React Native CLI.

También, se programaron 97 interfaces con sus distintos componentes. Más adelante, se realizó la programación del código fuente para los algoritmos que proporcionaron los cálculos para la estimación de biomasa y captura de carbono aéreo. Para terminar, se adaptó a la App para almacenar información dentro del teléfono móvil, se programaron los reportes y se visualizó la información en un mapa dinámico. Además, se programó el sitio web donde se puede obtener información a través de QGis. Como resultado de las etapas de diseño y producción en la Figura 3 se muestra la arquitectura del sistema híbrido basado en LAMJ+G.

La arquitectura que consta de tres secciones: cliente: donde los usuarios tienen comunicación con el sistema a través de los interfaces ya sea en una App en teléfonos móviles o a través de un sitio web accesible desde computadoras personales. Acceso web: Internet es la vía de comunicación de todos los elementos del sistema aprovechando las bondades del protocolo de comunicación TCP/IP la comunicación a las bases de datos, los distintos servicios web y el envío y recepción de archivos JSON para App. Servidor: computadora remota construida bajo la estructura LAMJ+G. El cliente puede acceder al sistema, a diversos recursos web y a distinto software haciendo peticiones al servidor. 


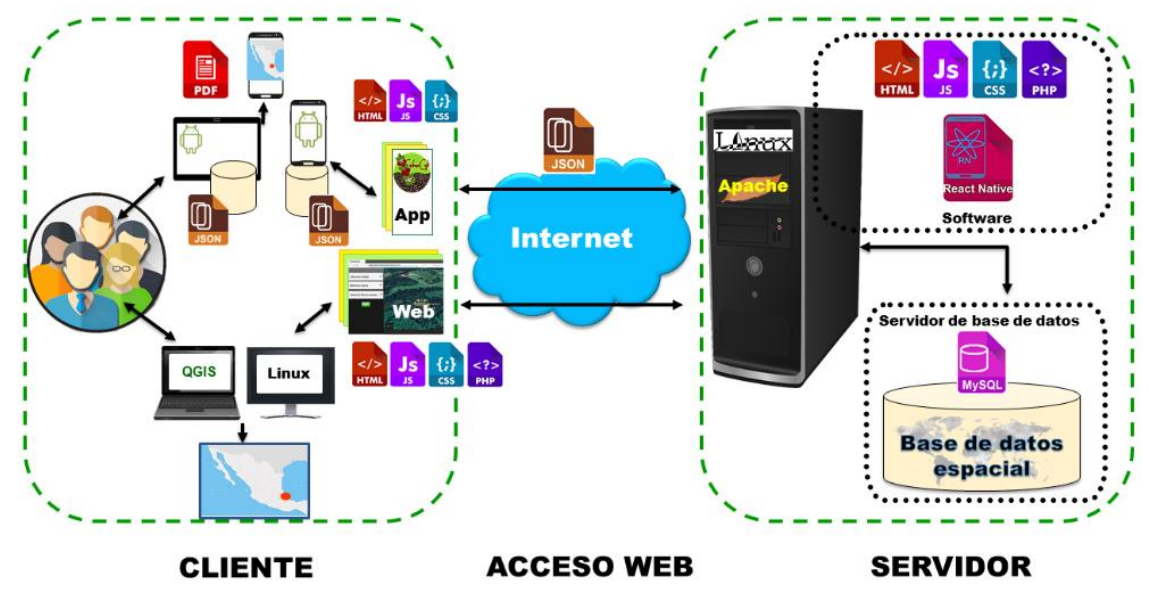

Figura 3. Arquitectura del sistema basada en web services usando LAMJ+G.

También, el servidor funciona como repositorio de la base de datos relacional que contiene la información de los usuarios, los sitios de muestreo, las georreferencias y los cálculos de retención de carbono. Además, permite el acceso de datos desde el QGis para analizar la información espacial. Asimismo, se diseñó el mapa de navegación y los permisos para cada usuario (Figura 4).

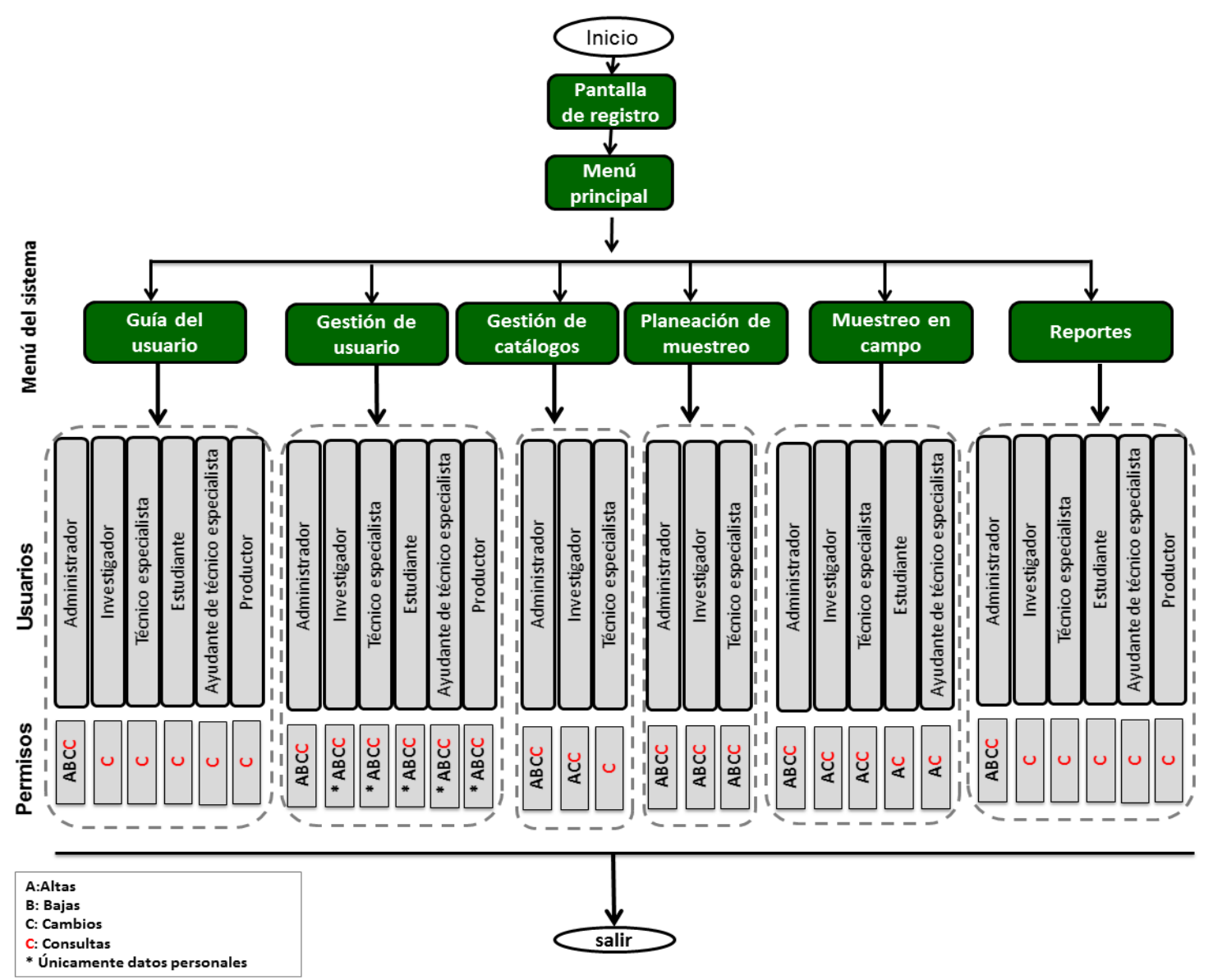

Figura 4. Mapa de navegación del sistema y permisos de usuario. 
Para que un usuario entre al sistema debe estar registrado, en caso contrario, debe llenar un formulario con sus datos. Más adelante, se dirige al menú principal. El usuario solo puede acceder a las secciones del menú y a algunas funciones de acuerdo con sus permisos asignados del perfil de registro (profesor, investigador, estudiante, productor, etc.).

La información del registro de usuarios y los catálogos de los lugares de muestreo quedan registrados en bases de datos. Asimismo, los datos recabados in situ quedan almacenados en el teléfono móvil y pueden enviarse al sistema en cuanto exista una conexión de Internet. La Figura 5 muestra algunos de los interfaces realizados en el sistema y vistos en el teléfono celular.
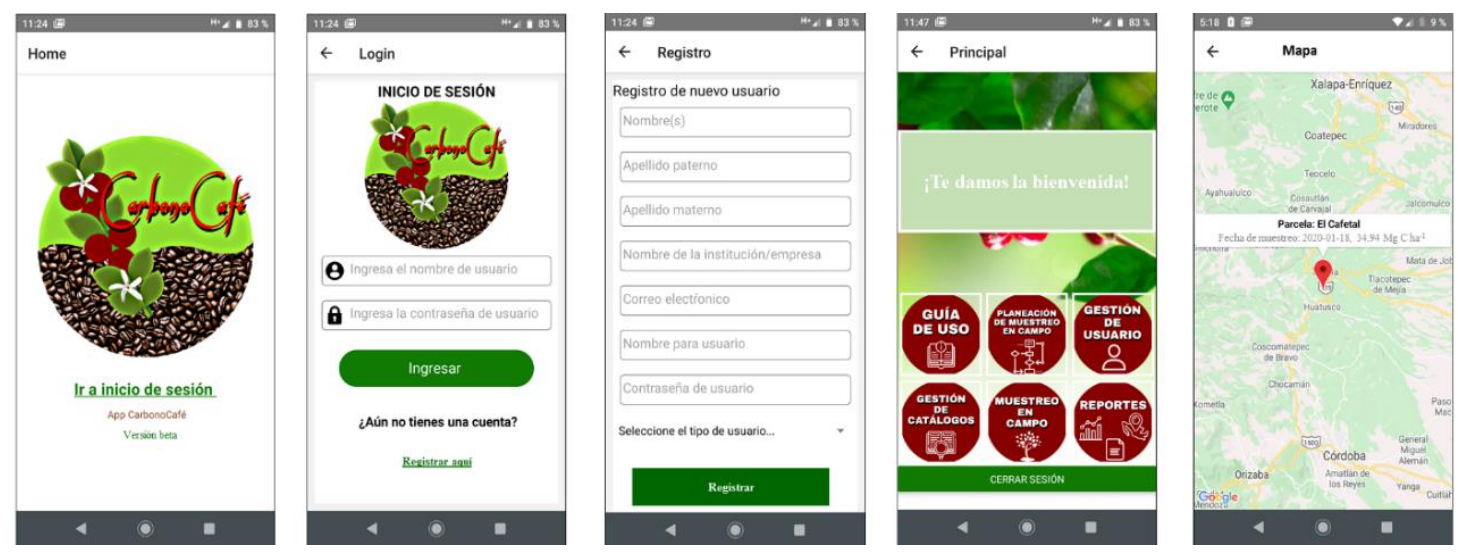

Figura 5. Interfaces de la App.

\section{Estabilización}

Se hicieron todas las pruebas del sistema utilizando datos obtenidos en campo en enero de 2020 con productores de Huatusco. Se hizo énfasis en el buen funcionamiento entre la base de datos relacional alojada en el servidor web remoto y la App, lo cual permitió buena comunicación entre el software, bases de datos, peticiones de reportes, mapas dinámicos, etc. Posterior a esto, se elaboraron los metadatos utilizando el modelo Dublin Core y se colocaron en la página principal del sitio web CarbonoCafé con dirección https://carbonocafe.tecnologiasgis.com.mx. Después, se realizaron las pruebas piloto entre el 22 de septiembre y el 05 de noviembre de 2020. Se instaló la App CarbonoCafé en diferentes teléfonos inteligentes. La instalación no tuvo ningún error y funciona adecuadamente.

\section{Evaluación}

Se evaluaron las etapas de planeación, análisis-diseño, producción-estabilización y mantenimientode acuerdo con lo establecido en la Figura 1 mediante rúbricas y cuestionarios elaborados para este sistema por los equipos multidisciplinarios.

\section{Mantenimiento}

Con las evaluaciones se detectaron errores, cambios o modificaciones en el sistema. Se siguieron las peticiones de los cuatro tipos de mantenimientos: correctivo, evolutivo, adaptativo y perfectivo. Primero se registró la petición del mantenimiento para diagnosticar el tipo de mantenimiento y se 
asignaron a los expertos responsables para cada proceso de la etapa de evaluación. Después, se analizó el problema detectado y se planteó la posible solución. Posterior a esto, se preparó la implementación de la modificación, donde se realizó un listado de prioridades, la secuencia para atenderlas y se estableció la fecha de entrega y el costo. Por último, se realizó el seguimiento y evaluación de los cambios hasta la aceptación por parte del equipo de expertos.

Cómo discusión se quiso contrastar el sistema CarbonoCafé contra otras iniciativas. CBP es una aplicación móvil para cuantificar carbono orgánico del suelo en Estados Unidos de América que almacena información en una base de datos manejada a través de un sistema web (Chotte et al., 2019). La aplicación desarrollada en esta investigación trabaja también tanto con una App como con un sistema Web, pero con un enfoque diferente ya que se calcula el carbono orgánico aéreo en especial en cultivos de café, pero para un sitio específico de México.

$\mathrm{CO}_{2}$ Land basado en $\mathrm{CO}_{2}$ FIX que trabaja bajo diferentes sistemas operativos que estima el carbono capturado y permite determinar anualmente sus cambios usando Sistemas de Información Geográficos (SIG) en distintos países (Ruiz-Guevara et al., 2020). El sistema de esta investigación trabaja con un sistema Web con datos georreferenciados, además, calcula la biomasa y el carbono orgánico aéreo a través de una App, la información se envía al servidor web cuando se tiene acceso a una conexión de Internet.

De acuerdo con lo anterior, se demostró que CarbonoCafé es diferente a los sistemas descritos, ya que este está enfocado a la estimación de captura de carbono aéreo en agroecosistemas de café de Huatusco, Veracruz, utiliza ecuaciones alométricas para estimar biomasa en especies específicamente de la región y se basa en la metodología de cuantificación de carbono en sistemas agroforestales de café bajo sombra propuestas por Tinoco y Escamilla (2017); RojasGarcía et al. (2015).

\section{Conclusiones}

El objetivo general de este trabajo se cumplió. La hipótesis no se rechaza porque se desarrolló un sistema computacional bajo una metodología híbrida llamada PADPEEM. El sistema CarbonoCafé será de impacto en la reducción de recursos humanos, económicos y de tiempo, para el cálculo de carbono aéreo in situ que además permite tener bases de datos que se pueden consultar. La información presentada en los reportes será de utilidad para mejorar la calidad de vida de los productores de café, debido que, aunque actualmente no ha trascendido en su totalidad el mercado de bonos de carbono, esta información puede ser utilizada por las organizaciones de productores de café para evidenciar de manera técnica y formal la estimación de carbono que capturan sus parcelas y así aspirar a pagos por servicios ambientales.

\section{Literatura citada}

Amaya, Y. D. 2013. Metodologías ágiles en el desarrollo de aplicaciones para dispositivos móviles. Estado Actual. Colombia. Rev. Tecnol. 12(2):111-123. Doi: http://dx.doi.org/10.18270/ rt.v12i2.1291.

Bakht. H. 2018. Mobile ad-hoc networking. South carolina. USA. BookSurge Publishing. 236 p. 
Bazán, P.; Fernández, A.; Rio, N.; Molinari, L.; Pérez, J. y Banchoff, M. 2017. Aplicaciones, servicios y procesos distribuidos: una visión para la construcción del software. $1^{\mathrm{a}}(\mathrm{Ed}$.). Buenos Aires, Argentina. Universidad de La Plata. 125 p.

Chotte, J. L.; Aynekulu, E.; Cowie, A.; Campbell, E.; Vlek, P.; Lal, R.; Kapović-Solomun, M.; Von Maltitz, G.; Kust, G.; Barger, N.; Vargas, R. y Gastrow, S. 2019. Aprovechar los beneficios del carbono de las prácticas de gestión sostenible de las tierras. Un informe de la interfaz ciencia-política. United Nations Convention to Combat Desertification (UNCCD). Bonn, Germany. 108 p.

Isaza, C. y Cornejo, J. M. 2015. Café y el ciclo del carbono. Cambio climático y carbono en café. Bogotá: Colombia. 40 p. https://www.agrolearning.com/educacion/file.php/124/ materialestudio/cafeyciclocarbono.pdf.

Meráz, J. M.; García Cué, J. L.; Fernández, Y. M.; Jiménez, M. A.; Medina, R. C. y SangermanJarquín, D. M. 2019. Elaboración de objetos de aprendizaje abiertos para ciencias agrícolas bajo la metodología PADDIEM. México. Rev. Mex. Cienc. Agríc. 10(5):1097-1111. Doi: https://doi.org/10.29312/remexca.v10i5.1701.

Rojas, F.; De-Jong, B. H. J.; Martínez P. y Paz, F. 2015. Database of 478 allometric equations to estimate biomass for Mexican trees and forests. Estados Unidos. J. Annal. Forest Sci. 72:835-864. Doi: https://doi.org/10.1007/s13595-015-0456-y.

Ruiz-Guevara, N.; Delgado-Rodríguez, D. y Carrera, F. 2020. Tecnologías para el monitoreo de impactos y emisiones de carbono del aprovechamiento forestal y de la trazabilidad de la madera de bosques naturales en Latinoamérica y el Caribe. Washington, DC. USA- Banco Interamericano de Desarrollo (BID). $114 \mathrm{p}$.

Tinoco, J. A. y Escamilla, E. 2017. Cuantificación de carbono en sistemas agroforestales de café bajo sombra. Centro nacional de Investigación, Innovación y Desarrollo Tecnológico del Café. Centro Regional Universitario Oriente-Universidad Autónoma Chapingo (UACH). Texcoco, Estado de México. 25 p. 